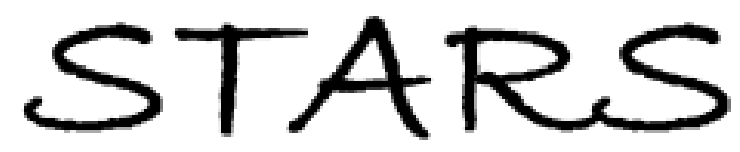

University of Central Florida

STARS

$1-1-2014$

\title{
Two- to one-dimensional crossover in graphene quantum dot arrays observed in reduced graphene oxide nanoribbons
}

Daeha Joung

University of Central Florida

Saiful I. Khondaker

University of Central Florida

Find similar works at: https://stars.library.ucf.edu/facultybib2010 University of Central Florida Libraries http://library.ucf.edu

This Article is brought to you for free and open access by the Faculty Bibliography at STARS. It has been accepted for inclusion in Faculty Bibliography 2010 s by an authorized administrator of STARS. For more information, please contact STARS@ucf.edu.

\section{Recommended Citation}

Joung, Daeha and Khondaker, Saiful I., "Two- to one-dimensional crossover in graphene quantum dot arrays observed in reduced graphene oxide nanoribbons" (2014). Faculty Bibliography 2010s. 5532. https://stars.library.ucf.edu/facultybib2010/5532

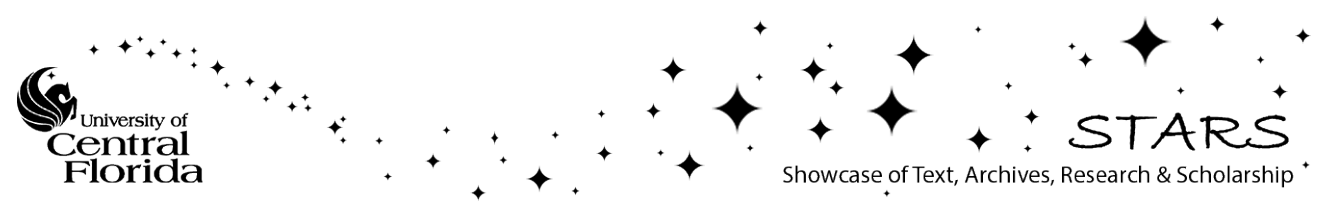




\title{
Two- to one-dimensional crossover in graphene quantum dot arrays observed in reduced graphene oxide nanoribbons
}

\author{
Daeha Joung ${ }^{1,2}$ and Saiful I. Khondaker ${ }^{1,2,3, *}$ \\ ${ }^{1}$ Nanoscience Technology Center, University of Central Florida, Orlando, Florida 32826, USA \\ ${ }^{2}$ Department of Physics, University of Central Florida, Orlando, Florida 32826, USA \\ ${ }^{3}$ School of Electrical Engineering and Computer Science, University of Central Florida, Orlando, Florida 32826, USA
}

(Received 31 December 2013; revised manuscript received 6 May 2014; published 9 June 2014)

\begin{abstract}
We investigate how the electron transport properties of graphene quantum dot (GQD) arrays transition from two dimensions (2D) to one dimension (1D) in lithographically defined reduced graphene oxide nanoribbons (RGONRs). From the low-temperature electron transport measurements of 200-, 100-, and 50-nm-wide RGONRs, we find that the energy barrier for charge transport increases with decreasing RGONR width in both the Coulomb blockade and the variable-range hopping regime. Different charge transport parameters for 200-nm RGONR are in agreement with 2D transport while these parameters show a gradual transition to 1D transport in 50-nm RGONR.
\end{abstract}

DOI: 10.1103/PhysRevB.89.245411

PACS number(s): 72.80.Vp, 72.20.Ee, 73.63.Kv

\section{INTRODUCTION}

Electronic transport properties of quantum dot (QD) arrays have attracted a lot of attention as they are a model system for the investigation of physics where quantum confinement and Coulomb charging energy $\left(E_{C}\right)$ play a significant role [1-17]. Transport properties of a QD array system are strongly influenced by dimensionality, as the charge transport pathways and energy requirement for the charge transport depends on the dimensions $[1,3,12,15-17]$. At sufficiently low temperatures, when the thermal energy $\left(k_{B} T\right)$ is lower than the $E_{C}$, the theory of QD array predicts that the current $(I)$ follows a scaling law with voltage $(V)$ above a threshold voltage $V_{t}[1]$,

$$
I \sim\left(\frac{V-V_{t}}{V}\right)^{\alpha}
$$

where the scaling exponent $\alpha$ depends on the dimensionality as the percolation of charges occur differently in different dimensions. The values of $\alpha$ is suggested to be $\sim 2$ for a twodimensional (2D) system, and $\sim 1$ for a one-dimensional (1D) system [1]. For $V<V_{t}$, a complete suppression of $I$ will occur due to a Coulomb blockade (CB) of charges as there is not enough thermal energy to overcome the $E_{C}$ of the QD array and the $V_{t}$ can be expressed as $[8,13,17]$

$$
V_{t}=V_{t 0}\left[1-\frac{4.8 k_{B} T}{E_{C} p_{C}}\right],
$$

where $p_{c}$ is the percolation threshold for the underlying lattice which depends on the dimension of the QD array system. At a fixed temperature $T$, the value of $V_{t}$ is expected to increase with decreasing dimension as the $p_{c}$ value is predicted to be $\sim 0.347$ in $2 \mathrm{D}$, while $p_{c}$ value is $\sim 1$ for $1 \mathrm{D}[8,13,17]$. The reason for this variation of $p_{c}$ is that in $2 \mathrm{D}$, the charge carriers have to overcome only $34 \%$ of all possible transport barriers to produce a current while in a truly $1 \mathrm{D}$ system, all the barriers must be overcome $[8,13,17]$. As the temperature is increased such that $k_{B} T>E_{C}$, the low-bias CB will be lifted, and one will enter the hopping transport regime. In such

*To whom correspondence should be addressed: saiful@ucf.edu a regime, the temperature dependence of resistance $(R)$ for a polydispersed QD array is expected to follow Efros-Shklovskii variable-range hopping (ESVRH) [18,19],

$$
R(T)=R_{0} \exp \left(\frac{T_{\mathrm{ES}}}{T}\right)^{1 / 2}, \text { with } T_{\mathrm{ES}}=\frac{C e^{2}}{4 \pi \varepsilon \varepsilon_{0} k_{B} \xi},
$$

where $R_{0}$ is a prefactor, $T_{\mathrm{ES}}$ is a characteristic temperature, $C$ is a numerical coefficient, $\varepsilon_{0}$ is the permittivity of vacuum, $\varepsilon$ is the dielectric constant of the material, and $\xi$ is localization length. The $T_{\mathrm{ES}}$ should increase with decreasing the dimensionality as the energy required for hopping transport increases when the dimension of the system decreases [17]. Since the $\xi$ depends on the material property and is independent of the dimension, $T_{\mathrm{ES}}$ can only increase if $C$ increases with dimension [20]. Theoretical simulation suggests that $C$ is $\sim 6.2$ for a $2 \mathrm{D}$ system [21]; however, no theoretical investigation of $C$ for $1 \mathrm{D}$ has been reported.

Experimentally, the transport properties of QD array in 2D have been extensively investigated in colloidal nanocrystals (NCs) of different sizes, shapes, interdot separations and disorders [3-8,16,17]. The CB and hopping transport have been reported and the study provided insight about charging energy, localization, and charge percolation due to structural and size induced disorders [3-8,16,17]. In contrast, only a limited number of studies was reported for (quasi-) 1D arrays $[3,12,22]$. However, a crossover from $2 \mathrm{D}$ to $1 \mathrm{D}$ in the same system remained elusive due to the challenges in fabricating samples of tunable dimensions. As a result, how the different charge transport parameters change, as the QD array dimension is tuned from to $2 \mathrm{D}$ to $1 \mathrm{D}$, remains largely unexplored. Recently, reduced graphene oxide (RGO), a chemically exfoliated graphene sheet, has emerged as a model system for studying 2D QD array where $s p^{2}$ graphene islands act as graphene quantum dots (GQDs) and $s p^{3}$ networks work as tunnel barriers [23,24]. The advantage of 2D RGO sheets is that they can be lithographically patterned and hence the RGO nanoribbon (RGONR) of different width can be fabricated and the dimensionality can be tuned. This may offer the opportunity to observe a crossover from 2D to 1D transport in a QD array system. 
Here, we demonstrate a crossover of electron transport from 2D GQD to 1D GQD array using lithographically patterned RGONRs. The 2D RGO sheets were assembled between prefabricated gold source and drain electrodes of $2 \mu \mathrm{m}$ channel length via dielectrophoresis (DEP), and RGONRs of 200-, $100-$, and 50-nm widths $(W)$ were lithographically fabricated. At low enough temperature, the $I-V$ curves of all RGONRs showed CB behaviors below a threshold voltage $\left(V_{t}\right)$. From the slope of the $V_{t}$ versus $T$ curve, we calculated a variation of $p_{c}$ from 0.46 for 200 -nm to 0.95 for 50 -nm RGONR, suggesting a decrease in the dimensionality of charge transport from $2 \mathrm{D}$ to $1 \mathrm{D}$ as the width of the RGONR decreases. For $V>V_{t}$, the $I$ follows a scaling behavior and the value of scaling exponent $\alpha$ decreases with RGONR width, consistent with the dimensional crossover behavior. In addition, temperaturedependent resistance of all RGONR followed ESVRH where $T_{\mathrm{ES}}$ increases due to an increase of $C$ with decreasing RGONRs width, which provides further evidence of a crossover from 2D to $1 \mathrm{D}$ charge transport.

\section{EXPERIMENTAL DETAILS}

RGONR devices used in this study were lithographically patterned from 2D RGO sheets. RGOs were obtained via chemical reduction of individual graphene oxide (GO). The average lateral dimension of the individual GO sheets was $\sim 0.8 \mu \mathrm{m}$ with a thickness of $\sim 1 \mathrm{~nm}$, indicating single-layer GO [25]. The RGO sheets were then produced via chemical reduction in hydrazine hydrate for $60 \mathrm{~min}$ at $90^{\circ} \mathrm{C}$. The carbon $s p^{2}$ fraction (or reduction efficiency) of RGO sheets used in this study was $\sim 80 \%$ calculated from the ratio of the integrated peak areas corresponding to the $\mathrm{C}-\mathrm{C}$ peak to the total area under the $\mathrm{C} 1 s$ spectrum from the $\mathrm{x}$-ray photoelectron spectroscopy (XPS) spectra (see Supplemental Material for details [26]). A mean size of $s p^{2}$ graphene domain (or GQD) is estimated to be $\sim 4.5 \mathrm{~nm}$ using the intensity ratio of the $D$ band to the $G$ band $\left(I_{D} / I_{G}\right)$ in Raman spectra (see Supplemental Material [27]). This is in excellent agreement with our previous report where the average GQD size of $4.18 \pm 0.6 \mathrm{~nm}$ was determined from the electron transport study of the same RGO $(80 \%$ $s p^{2}$ fraction) [24]. This value is also in good agreement with transmission electron microscopy (TEM) and scanning tunneling microscopy (STM) studies of RGO, where the size of the graphene domain was reported to vary from 3 to $10 \mathrm{~nm}$ [28]. More details about the synthesis and structure of RGO sheets can be found in our previous publications [23-25,29].

Devices were fabricated on heavily doped silicon $(\mathrm{Si})$ substrates capped with a thermally grown 250 -nm-thick $\mathrm{SiO}^{2}$ layer. Source and drain electrode patterns of $2 \times 1 \mu \mathrm{m}$ (channel length $\times$ width) were defined by electron beam lithography (EBL) followed by thermal deposition of 3-nm-thick $\mathrm{Cr}$ and 25-nm-thick Au. The RGO sheets were then assembled between the prefabricated source and drain electrodes using ac dielectrophoresis (DEP) [25]. The average two-terminal resistance of the assembled RGO devices was $\sim 2.5 \mathrm{M} \Omega$ from which we estimate a conductivity value to be $\sim 170 \Omega^{-1} \mathrm{~m}^{-1}$, in good agreement with reported conductivities of other RGO devices [23,25,30,31], suggesting that the contact resistance in our sample is not significant and has negligible effect in the transport study reported here. After the assembly, RGONRs of
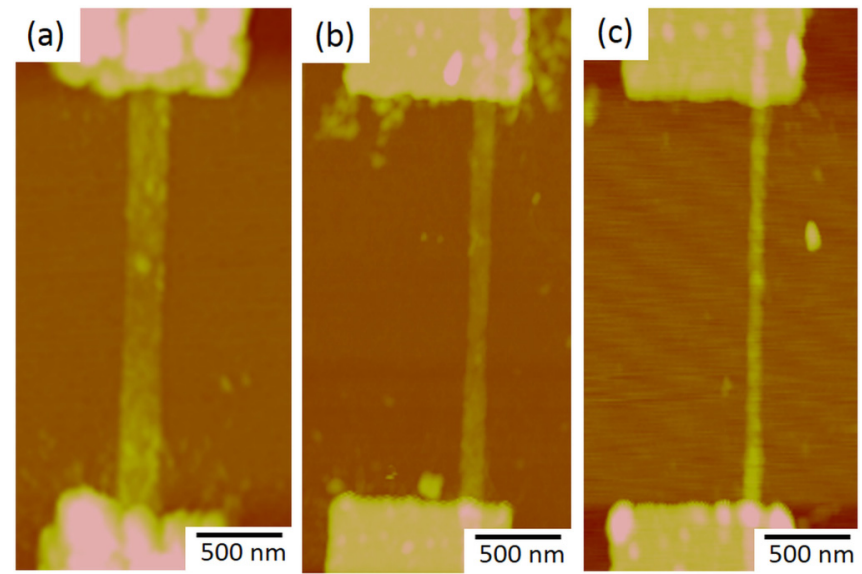

FIG. 1. (Color online) AFM images of RGONR with different widths of (a) $200 \mathrm{~nm}$, (b) $100 \mathrm{~nm}$, and (c) $50 \mathrm{~nm}$. The channel length is $2 \mu \mathrm{m}$.

widths 200,100, and $50 \mathrm{~nm}$ were patterned using the standard EBL process and oxygen plasma etching of the exposed RGO sheet. Subsequently, the devices were thermally annealed in argon:hydrogen $(1: 3)$ gas at $200^{\circ} \mathrm{C}$ for $1 \mathrm{~h}$. Figures 1(a)-1(c) show atomic force microscope (AFM) images of the 200-, 100-, and 50-nm RGONRs, respectively. The thickness of the RGONR varies from 2 to $5 \mathrm{~nm}$ across the channel. This is typical for all of the RGONRs in this study. The devices were, then, bonded to a chip carrier and loaded into a variable-temperature cryostat for temperature-dependent electronic transport measurements. The measurements were performed using a Keithley 2400 source meter and a current preamplifier (DL 1211) capable of measuring a picoampere signal interfaced with the LABVIEW program. We measured a total of six devices, two for each RGONR.

\section{RESULTS AND DISCUSSION}

Figure 2(a) shows the current-voltage $(I-V)$ characteristics of our best 50-nm RGONR device measured from 4.2 to $70 \mathrm{~K}$. Below $40 \mathrm{~K}$, all the $I-V$ curves show a complete suppression of currents below a threshold voltage $V_{t}$. This is a characteristic of a $\mathrm{CB}$ of charge transport as expected from the QD array model. At these temperatures, there is not enough thermal energy for the charge carriers to overcome the charging energy of the QDs $\left(k_{B} T>E_{C}\right)$ and the transport is blocked. A similar CB of charges was observed for 100- and 200-nm RGONR as well. This is shown in Fig. 2(b) where we plot the $I-V$ characteristics measured at $4.2 \mathrm{~K}$ for our best 200-, 100-, and 50 -nm RGONRs. The $I-V$ curves at other temperatures for 200- and 100-nm RGONR are presented in the Supplemental Material [32]. Although all of our RGONRs show a CB of charges consistent with the charge transport through the GQD array [23,24], a significant observation in Fig. 2(b) is that the threshold voltage (defined as the voltage below which a complete suppression of current is observed and indicated by an arrow for each $I-V$ curve) at $4.2 \mathrm{~K}$ increases with decreasing width. The measured $V_{t}$ values at $4.2 \mathrm{~K}$ were $1.22,2.24$, and $3.60 \mathrm{~V}$ (the error in determining $V_{t}$ is $\pm 0.02 \mathrm{~V}$, the voltage sweep step in our measurement) for 200-, 100-, and 50-nm 

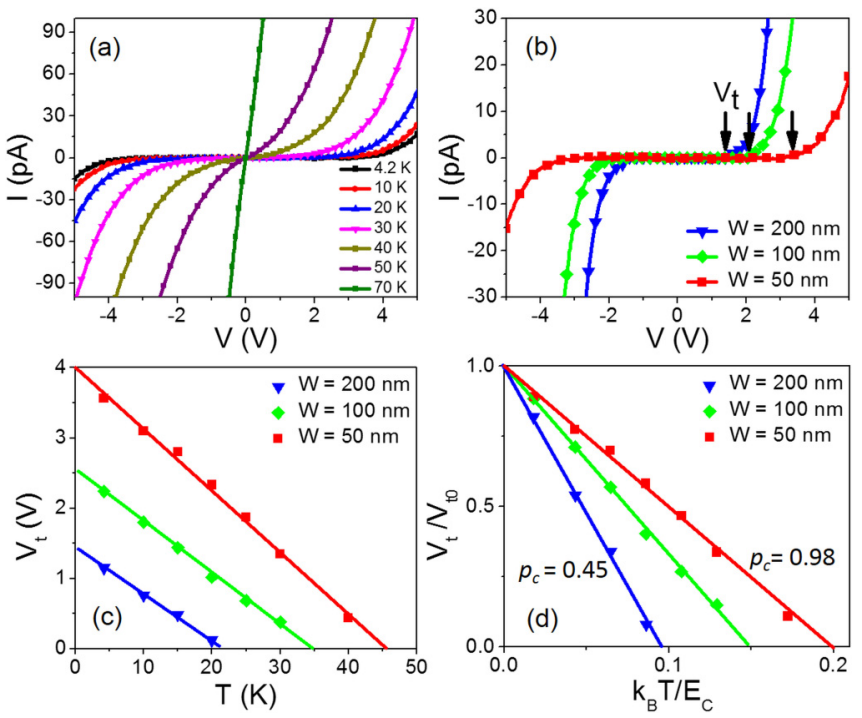

FIG. 2. (Color online) (a) Current-voltage ( $I-V)$ characteristics of a 50-nm-wide RGONR at different temperatures from 4.2 to $70 \mathrm{~K}$. Below $40 \mathrm{~K}, I$ became zero for $V<V_{t}$ due to Coulomb blockade (CB) of charges. (b) $I$ - $V$ characteristics of 200-, 100-, and 50-nm-wide RGONRs at $4.2 \mathrm{~K}$. The $V_{t}$ values (indicated by arrows) increase with decreasing RGONR width. (c) $V_{t}$ plotted versus $T$ for different RGONR widths. (d) Normalized threshold, $V_{t} / V_{t 0}$ versus effective temperature variable $k_{B} T / E_{C}$ for all RGONRs. The slope is $4.8 / p_{C}$.

RGONRs, respectively. In a QD array system, the values of $V_{t}$ mainly depend on the size of the QD, interdot separation, and dimension of the system. For a RGO sheet, the QD size and interdot separation depends on the $s p^{2}$ fraction of the sheet [24]. In this study, we fabricated RGONR from the same $s p^{2}$ fraction sheet, so all the RGONR devices should have the same average QD size and interdot separation. This is further confirmed by comparing the $V_{t}$ values of our 200-nm RGONR of $2 \mu \mathrm{m}$ channel length with our previously reported (Ref. [23]) 2D RGO devices of $0.5 \mu \mathrm{m}$ channel length. According to the CB model, the values of $V_{t}$ should scale with the number of QDs in the transport path provided that the dimensionality of the array and the QD size remain unchanged [23]. In Ref. [23], $V_{t}$ at $4.2 \mathrm{~K}$ was 0.28 while it is 1.2 (approximately four times higher) for the 200-nm-wide RGONR device in this study, consistent with the CB model. In the present study, all the RGONR devices have the same channel length $(2 \mu \mathrm{m})$. Therefore, the increase of $V_{t}$ with reducing width is indicative of the effects of dimensionality on the charge transport. Increase of $V_{t}$ with reducing width suggests that the energy requirement to overcome the $\mathrm{CB}$ of charges through the GQD array increases with decreasing width of RGONR. The increasing energy requirement with reducing dimension is expected from the QD array model. Therefore, our results suggest a dimensional crossover with the reduction of RGONR width.

The increase of $V_{t}$ with decreasing NR width were seen at all temperatures as shown in Fig. 2(c). In addition, we see that the $V_{t}$ values linearly decreased with increasing $T$ for all RGONRs as expected from Eq. (2). From these curves, we can determine the intercepts $V_{t 0}$ and replot the $V_{t} / V_{t 0}$ data as a function of $k_{B} T / E_{C}$ as shown in Fig. 2(d). The slope of each curve is $4.8 / p_{c}$ from which we obtain the $p_{c}$ values as $0.45,0.68$, and 0.98 for 200-, 100-, and 50-nm RGONRs, respectively. In plotting Fig. 2(d) we used an $E_{C}$ value of $20 \mathrm{meV}$ calculated using a GQD size $4.5 \mathrm{~nm}$ determined from the Raman spectra [33]. The other devices yield $p_{c}$ values of 0.47, 0.72, and 0.92 (see Supplemental Material [34]) giving average $p_{c}$ values of $0.46 \pm 0.01,0.70 \pm 0.02,0.95 \pm 0.03$ for 200-, 100-, and 50-nm RGONRs, respectively. A $p_{c}$ value of 0.347 is expected for a $2 \mathrm{D}$ system while it is expected to be 1 for a $1 \mathrm{D}$ system $[8,13,17]$. This suggests that the transport of our 200-nm RGONR is in fairly good agreement with 2D while the transport of 50-nm RGONR is in close agreement with 1D transport. It is interesting to note that the transition from 2D to 1D transport is not an abrupt one; rather it is a gradual transition. The reason for such a gradual transition is not clear to us and more theoretical and experimental study will be needed to elucidate on this.

The dimensional crossover is further confirmed by analyzing the scaling behavior of $I-V$ curves for $V>V_{t}$. As discussed in Eq. (1), the theory of a QD array predicts that at sufficiently low temperatures $I$ follows a scaling behavior for $V>V_{t}$ where the scaling exponent $\alpha$ depends on the dimensionality of the QD array system. Figures 3(a)-3(c) show $I$ plotted versus $\left(V-V_{t}\right) / V_{t}$ in a log-log scale at $4.2 \mathrm{~K}$ for $200-$, 100-, and 50-nm RGONRs, respectively. The symbols are the experimental data points while the solid lines are fits to the Eq. (1). It can be clearly seen that for all the RGONRs, the data follow Eq. (1). From the best straight line fits, we obtained the values of $\alpha$ to be $3.14 \pm 0.03,2.82 \pm 0.03$, and $1.61 \pm$ 0.02 for $200-, 100-$, and $50-n m$ RGONRs, respectively. The $\alpha$ values of the other set of RGONRs showed $3.32 \pm 0.02$,
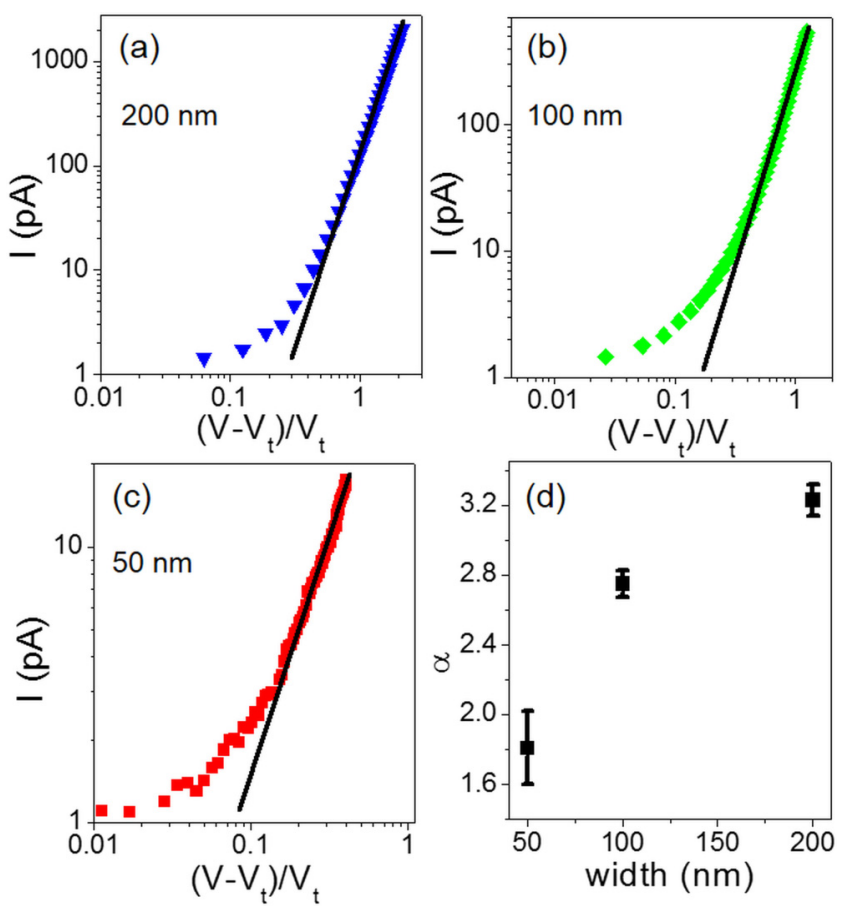

FIG. 3. (Color online) $I$ versus $\left(V-V_{t}\right) / V_{t}$ curves plotted in a $\log$ - $\log$ scale at $4.2 \mathrm{~K}$ for (a) 200-, (b) 100-, and (c) 50-nm-wide RGONRs. The symbols are the experimental data points, while the solid lines are fit to Eq. (1). (d) Average scaling exponent $\alpha$ versus width of RGONRs. 
$2.67 \pm 0.03$, and $2.02 \pm 0.03$ [34]; hence we obtain average $\alpha$ values of $3.23 \pm 0.09,2.75 \pm 0.08$, and $1.81 \pm 0.21$ for 200-, 100-, and 50-nm RGONRs, respectively. Figure 3(d) shows a plot of average $\alpha$ versus RGONR width from which we can clearly see a decrease of $\alpha$ with decreasing RGONR width. In previous experimental studies of $2 \mathrm{D}$ homogeneous metal nanocrystal arrays the values of $\alpha$ were reported to be in the range of 2-2.5 [3-6,8,16]. However, it has been reported that when a 2D QD array is inhomogeneous and contains topological defects, the values of $\alpha$ can be as high as 4.0 [35]. Since RGO contains a lot of topological defects, the $\alpha$ values for a $2 \mathrm{D}$ RGO sheet were reported to be $\sim 3.2-3.4$ [23,24]. Therefore, $\alpha \sim 3.2$ for the 200-nm RGONR is consistent with a 2D GQD array with topological defects. On the other hand, the value of $\alpha=1$ for a 1D system has not been experimentally validated yet. A graphitic carbon 1D nanoparticle chain showed $\alpha \sim 1.3$ while a (quasi-) $1 \mathrm{D}$ metal nanoparticle showed $\alpha \sim 1.4-1.6[3,12,22]$. We therefore believe that $\alpha \sim 1.8$ for $50-\mathrm{nm}$ RGONR is reasonable for a 1D GQD array considering topological disorders.

We have also studied the dimensional crossover behavior in the VRH regime. Figure 2(a) shows that above $40 \mathrm{~K}$, the $\mathrm{CB}$ is lifted and there is a low-bias Ohmic regime from which we can determine the resistance $R$ as a function of temperature $T$. Figure 4(a) shows a semilog scale plot of $R$ versus $T$ for all the RGONR devices. It can be seen that the $R$ for all of the RGONRs varied about three orders of magnitude with temperature. Such a large variation is important for accurate analysis of VRH conduction. Figure 4(b) shows a semilog scale plot of $R$ versus $T^{-1 / 2}$ for all the RGONR devices. The symbols are the experimental points and the solid lines are a fit to $T^{-1 / 2}$ behavior. As expected from Eq. (3), the data for all the samples fit very well with $T^{-1 / 2}$ behavior. From the slopes of Fig. 4(b), we obtained the characteristic temperature $T_{\mathrm{ES}}$ for all of the RGONRs. The $T_{\mathrm{ES}}$ values were increased with decreasing width of RGONRs. The values of $T_{\mathrm{ES}}$ are 5400, 7500, and $11000 \mathrm{~K}$ for 200-, 100-, and 50-nm RGONRs, respectively. In our previous study of 2D ESVRH done on this RGO sheet (carbon $s p^{2}$ fraction of $\sim 80 \%$ ), we obtained $\xi \sim 3 \mathrm{~nm}[23,29]$. By using the value of $\xi$ in Eq. (3), we obtain the values of $C$ as 3.3, 4.7, and 6.8 for 200-, 100-, and 50-nm RGONRs, respectively. The other RGONR devices showed the $C$ values of 3.7, 5.1, and 8.6 [34] giving average $C$ values of $3.5 \pm 0.2,4.9 \pm 0.2$, and $7.7 \pm 0.9$ for $200-, 100$-, and 50-nm RGONRs, respectively. Figure 4(b) inset shows a plot of $C$ versus RGONR width from which we can clearly see an increase of $C$ with decreasing RGONR width. Although the values of $C$ are expected to be 6.2 in 2D [21], experimentally a $C$ value of 2.8 was used in RGO, functionalized graphene, and graphene antidot lattices [23,29,36]. Therefore, the value of $C=3.5$ in 200-nm RGONR is in close agreement with hopping transport in $2 \mathrm{D}$ disordered graphene. While theoretical investigations of $C$ in 1D QD array systems need to be made, our study suggest that $C$ should be $\sim 7.7$ in a 1D GQD array. The increasing $C$ with decreasing width of RGONR is consistent with the dimensional crossover in RGONRs.

In the following, we discuss the crossover in terms of different relevant length scales of our devices. If the average size of the GQDs is $r$, the interdot separation is $d$, and the width of RGONR is $W$, then one expects a perfect $1 \mathrm{D}$ conduction for $W \sim r+d$ and 2D conduction for $W \gg r+d$. For the study presented here, the average values for $r$ and $d$ are 4.5 and $3.0 \mathrm{~nm}$, respectively [24], for the RGO (80\% $s p^{2}$ fraction) used in fabricating all the devices. Therefore, in our study, $W \sim 7(r+d)$ for 50-nm RGONR and $W \sim 27(r+d)$ for 200-nm RGONR. Theoretical study of charge transport of a QD array with varying QD-QD junction sizes have shown the scaling exponent $\alpha=1$ (expected for 1D) can be obtained for five junctions along the width [37]. This study showed that the values of $\alpha$ increase with increasing the junction sizes and saturate to 2.3 (expected for 2D) at 26 junctions. In our study, the average junction size is about 7 for $50-\mathrm{nm}$ RGONR where we observed 1D transport, in close agreement with Ref. [37]. In addition, we note that the GQDs in our study are strongly inhomogeneous with a maximum domain size of up to $10 \mathrm{~nm}$ possible, as has been seen from the TEM and STM studies [28]. In addition, the RGO contains topological defect, so, although our $W$ for 50 -nm RGONR is larger than the average $r+d$, we believe that the inhomogeneity and topological defects can create a $1 \mathrm{D}$ bottleneck for charge transport rendering the 50-nm RGONR to behave like 1D. In addition, the strong inhomogeneity creates significant charge disorder making the
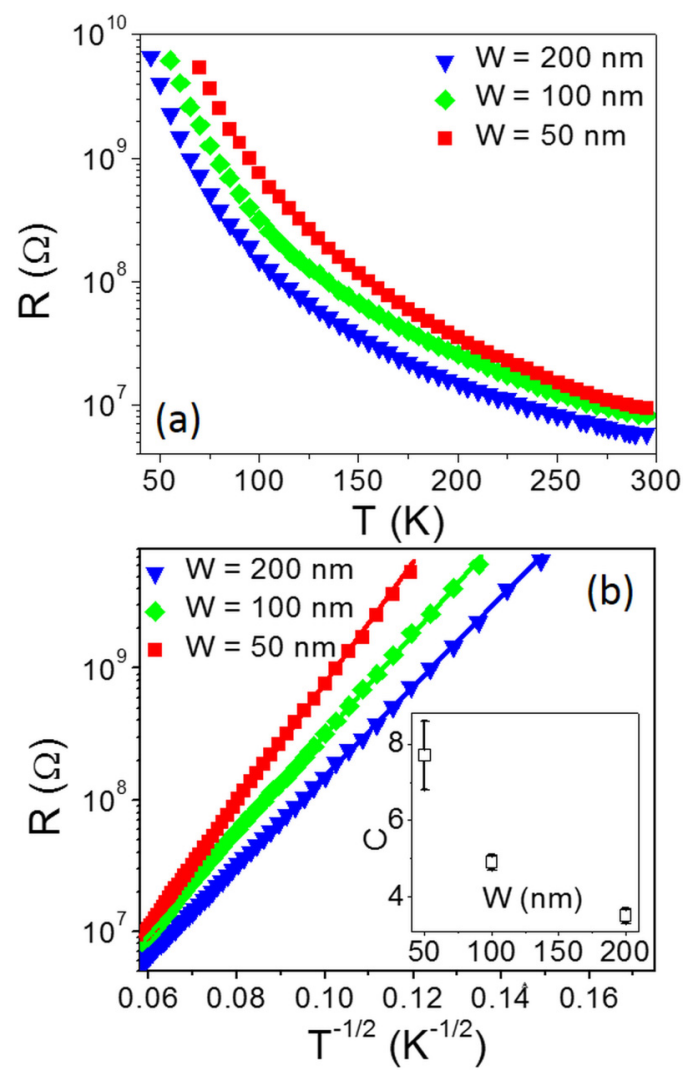

FIG. 4. (Color online) (a) Semilog scale plot of resistance versus temperature $(R-T)$ for 200-, 100-, and 50-nm wide RGONRs. (b) Semilog scale plot of $R$ versus $T^{-1 / 2}$ for all RGONRs. The symbols are the experimental points and the solid lines are a fit to $T^{-1 / 2}$ behavior. From the slopes, $T_{\mathrm{ES}}=5400,7500$, and $11000 \mathrm{~K}$ were obtained for 200-, 100-, and 50-nm-wide RGONRs, respectively. Inset: Average numerical coefficient $C$ versus width $(W)$ of RGONRs. 
realization of 1D transport easier. As the energy costs for tunneling through smaller GQDs are higher, the charge carrier will choose the path that costs the minimum energy (larger size of GQD). Therefore, even if there is more than $1 \mathrm{GQD}$ along the width, the charge will transport through the larger size GQD effectively making $1 \mathrm{D}$ transport. The probability for creating such a 1D transport bottleneck decreases with increasing the width of the RGONR as there will be several preferable charge transport pathways to choose from. As a result, the 200-nm RGONR shows a 2D transport. Interestingly, our 200-nm RGONR has 27 junctions, just above the threshold for 2D transport suggested by the theoretical study (Ref. [37]).

\section{CONCLUSION}

In conclusion, we provided insight into the intriguing transport properties of low-dimensional graphene based devices. We demonstrated that a crossover of electron transport from a 2D GQD array to a 1D GQD array occurs in lithographically defined RGONRs as the width decreases from 200 to $50 \mathrm{~nm}$.
At low enough temperature, there is a complete suppression of current below a $V_{t}$ which increases with decreasing RGONR width. From the slope of the $V_{t}$ versus $T$ curve, we calculated a variation of $p_{c}$ from 0.46 for $200-\mathrm{nm}$ to 0.95 for $50-\mathrm{nm}$ RGONR, consistent with a crossover of charge transport from 2D to 1D. For $V>V_{t}$, the $I$ follows a scaling behavior with a decrease of scaling factor $\alpha$ from 3.2 to 1.8 with decreasing RGONR width, suggesting a decrease of dimensionality. Finally, temperature-dependent resistance of all RGONRs followed ESVRH where $T_{\mathrm{ES}}$ increases due to an increase of $C$ from 3.5 to 7.7 with decreasing RGONRs width, which provides further evidence of a crossover from 2D to 1D charge transport. Our results show a clear experimental evidence for a crossover from 2D to 1D charge transport in a GQD array in graphene based materials.

\section{ACKNOWLEDGMENT}

This work has been partially supported by US NSF under Grant No. ECCS 0748091 to S.I.K.
[1] A. A. Middleton and N. S. Wingreen, Phys. Rev. Lett. 71, 3198 (1993).

[2] C. A. Stafford and S. Das Sarma, Phys. Rev. Lett. 72, 3590 (1994).

[3] A. J. Rimberg, T. R. Ho, and J. Clarke, Phys. Rev. Lett. 74, 4714 (1995).

[4] C. T. Black, C. B. Murray, R. L. Sandstrom, and S. Sun, Science 290, 1131 (2000).

[5] R. Parthasarathy, X.-M. Lin, and H. M. Jaeger, Phys. Rev. Lett. 87, 186807 (2001).

[6] M. G. Ancona, W. Kruppa, R. W. Rendell, A. W. Snow, D. Park, and J. B. Boos, Phys. Rev. B 64, 033408 (2001).

[7] K. C. Beverly, J. F. Sampaio, and J. R. Heath, J. Phys. Chem. B 106, 2131 (2002).

[8] R. Parthasarathy, X.-M. Lin, K. Elteto, T. F. Rosenbaum, and H. M. Jaeger, Phys. Rev. Lett. 92, 076801 (2004).

[9] J. Zhang and B. I. Shklovskii, Phys. Rev. B 70, 115317 (2004); B. Skinner, T. Chen, and B. I. Shklovskii, ibid. 85, 205316 (2012); T. Chen, B. Skinner, and B. I. Shklovskii, Phys. Rev. Lett. 109, 126805 (2012).

[10] D. Yu, C. Wang, B. L. Wehrenberg, and P. Guyot-Sionnest, Phys. Rev. Lett. 92, 216802 (2004).

[11] P. Beecher, A. J. Quinn, E. V. Shevchenko, H. Weller, and G. Redmond, J. Phys. Chem. B 108, 9564 (2004).

[12] K. Elteto, X.-M. Lin, and H. M. Jaeger, Phys. Rev. B 71, 205412 (2005).

[13] H. E. Romero and M. Drndic, Phys. Rev. Lett. 95, 156801 (2005).

[14] A. Zabet-Khosousi, P.-E. Trudeau, Y. Suganuma, A.-A. Dhirani, and B. Statt, Phys. Rev. Lett. 96, 156403 (2006).

[15] I. Beloborodov, A. Lopatin, V. Vinokur, and K. Efetov, Rev. Mod. Phys. 79, 469 (2007).

[16] A. Zabet-Khosousi and A. A. Dhirani, Chem. Rev. 108, 4072 (2008).

[17] K. Xu, L. Qin, and J. R. Heath, Nat. Nanotechnol. 4, 368 (2009).
[18] A. L. Efros and B. I. Shklovskii, J. Phys. C: Solid State Phys. 8, L49 (1975).

[19] B. I. Shklovskii and A. L. Efros, Electronic Properties of Doped Semiconductors (Springer, Berlin, 1984).

[20] M. Fogler, S. Teber, and B. Shklovskii, Phys. Rev. B 69, 035413 (2004).

[21] V. L. Nguyen, Sov. Phys. Semicond. 18, 207 (1984).

[22] A. Bezryadin, R. M. Westervelt, and M. Tinkham, Appl. Phys. Lett. 74, 2699 (1999).

[23] D. Joung, L. Zhai, and S. I. Khondaker, Phys. Rev. B 83, 115323 (2011).

[24] D. Joung and S. I. Khondaker, J. Phys. Chem. C 117, 26776 (2013).

[25] D. Joung, A. Chunder, L. Zhai, and S. I. Khondaker, Nanotechnology 21, 165202 (2010).

[26] See Supplemental Material at http://link.aps.org/supplemental/ 10.1103/PhysRevB.89.245411 for XPS spectra of RGO sheets and calculations of carbon $s p^{2}$ fractions.

[27] An estimate of graphene domain size can be obtained by comparing the intensity of $D$ and $G$ peaks of the Raman spectrum of the RGO sheets using the Tunistra-Koening relation $[\mathrm{F}$. Tuinstra and J. L. Koenig, J. Chem. Phys. 53, 1126 (1970)]. We obtained a mean domain size of $\sim 4.5 \mathrm{~nm}$ from our Raman data See Supplemental Material at http://link.aps.org/supplemental/ 10.1103/PhysRevB.89.245411 for Raman spectra of RGO sheets.

[28] K. Erickson, R. Emi, Z. Lee, N. Alem, W. Gannett, and A. Zettl, Adv. Mater. 22, 4467 (2010); C. Gómez-Navarro, J. C. Meyer, R. S. Sundaram, A. Chuvilin, S. Kurasch, M. Burghard, K. Kern, and U. Kaiser, Nano Lett. 10, 1144 (2010).

[29] D. Joung and S. I. Khondaker, Phys. Rev. B 86, 235423 (2012).

[30] C. Gómez-Navarro, R. T. Weitz, A. M. Bittner, M. Scolari, A. Mews, M. Burghard, and K. Kern, Nano Lett.7, 3499 (2007).

[31] I. Jung, D. A. Dikin, R. D. Piner, and R. S. Ruoff, Nano Lett.8, 4283 (2008). 
[32] See Supplemental Material at http://link.aps.org/supplemental/ 10.1103/PhysRevB.89.245411 for temperature-dependent $I-V$ curves of 200- and 100-nm RGONRs.

[33] We obtained a mean domain size of $\sim 4.5 \mathrm{~nm}$ from our Raman data (see Ref. [27]). Using $E_{C} \approx e^{2} / 2 C_{\Sigma}$, we calculate the $E_{C}$ of the GQDs. $C_{\Sigma}$ can be estimated from the geometrical consideration and can be written as $C_{\Sigma}=C_{g}+9 C$, where $C_{g} \approx 4 \pi \varepsilon \varepsilon_{0} r$ and $C \approx 2 \pi \varepsilon \varepsilon_{0} r \ln [(r+d) / d]$ are self-capacitance and mutual capacitance of QDs, respectively. We use an interdot spacing value $d$ of $1.5 \mathrm{~nm} . E_{C} \sim 20 \mathrm{meV}$ has been calculated. See also Refs. [23,24].

[34] See Supplemental Material at http://link.aps.org/supplemental/ 10.1103/PhysRevB.89.245411 for $V_{t}$ versus $T, V_{t} / V_{t 0}$ versus
$k_{B} T / E_{c}, I$ versus $\left[\left(V_{t}-V\right) / V_{t}\right]$, and $\ln R$ versus $T^{-1 / 2}$ plots of other RGONRs.

[35] M. O. Blunt, M. Šuvakov, F. Pulizzi, C. P. Martin, E. PauliacVaujour, A. Stannard, A. W. Rushforth, B. Tadić, and P. Moriarty, Nano Lett. 7, 855 (2007).

[36] A. Pachoud, M. Jaiswal, Y. Wang, B.-H. Hong, J.-H. Ahn, K. P. Loh, and B. Özyilmaz, Sci. Rep. 3, 3404 (2013); H. Zhang, E. Bekyarova, J.-W. Huang, Z. Zhao, W. Bao, F. Wang, R. C. Haddon, and C. N. Lau, Nano Lett. 11, 4047 (2011); A. J. M. Giesbers, E. C. Peters, M. Burghard, and K. Kern, Phys. Rev. B 86, 045445 (2012).

[37] R. W. Rendell and M. G. Ancona, IEEE Trans. Nanotechnol. 2, 75 (2003). 